\title{
THE NILPOTENCY CLASS OF FINITE GROUPS OF EXPONENT $p$
}

\author{
MICHAEL VAUGHAN-LEE
}

\begin{abstract}
We investigate the properties of Lie algebras of characteristic $p$ which satisfy the Engel identity $x y^{n}=0$ for some $n<p$. We establish a criterion which (when satisfied) implies that if $a$ and $b$ are elements of an Engel- $n$ Lie algebra $L$ then $a b^{n-2}$ generates a nilpotent ideal of $L$. We show that this criterion is satisfied for $n=6, p=7$, and we deduce that if $G$ is a finite $m$-generator group of exponent 7 then $G$ is nilpotent of class at most $51 \mathrm{~m}^{8}$.
\end{abstract}

\section{INTRODUCTION}

In 1989 E. I. Zelmanov [18, 19] solved the restricted Burnside problem by proving that for every positive integer $m$ and every prime-power $p^{k}$ there is a bound on the possible orders of finite $m$-generator groups of exponent $p^{k}$. Together with results of Hall and Higman [3], this proves that for all positive integers $m$ and $n$ there is a bound on the possible orders of finite $m$-generator groups of exponent $n$. Nevertheless, the problem of obtaining explicit bounds remains open in most cases. If $G$ is a finite $m$-generator group of exponent $p^{k}$ then $G$ is nilpotent, and in some ways it is more natural to look for bounds on the nilpotency class of $G$, rather than to look directly for bounds on the order of $G$. If we can show that the class of $G$ is bounded by $c$, say, then it is easy to see that the order of $G$ is at most $p^{k . m^{c}}$. However, neither Zelmanov's solution of the restricted Burnside problem for prime-power exponent, nor Kostrikin's 1959 solution of the problem for prime exponent [8], give explicit bounds for either the order or the class of $G$. Adjan and Razborov [1] gave the first primitive recursive bound for the class of a finite $m$-generator group of exponent $p$, and similar bounds may be found in [9]. Vaughan-Lee and Zelmanov [17] showed that if $G$ is a finite $m$-generator group of exponent $p$ then the class of $G$ is at most

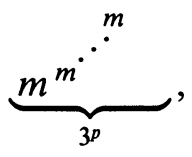

and they showed that if $G$ is a finite $m$-generator group of prime-power expo-

Received by the editors February 10, 1994.

1991 Mathematics Subject Classification. Primary 20D15, 20F12, 20F18; Secondary 20F40, 20F45, 20F50. 
nent $q$ then the class of $G$ is at most

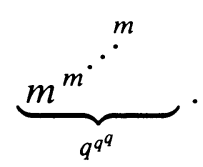

It should be possible to improve these bounds considerably, and in special cases we can do much better. Groups of exponent 2 are abelian, and groups of exponent 3 are nilpotent of class at most 3 (Levi and van der Waerden [10]). Groups of exponent 4 are locally finite (Sanov [12]), and it follows from results of Gupta and Newman [2] and Razmyslov [11] that $m$-generator groups of exponent 4 have class at most $3 m-2$. Higman [7] solved the restricted Burnside problem for exponent 5 in 1956, and he showed that there is a positive integer $N$ such that if $G$ is a finite $m$-generator group of exponent 5 then $G$ is nilpotent of class at most $N m$. Higman did not compute an explicit value for $N$, but Havas, Newman, and Vaughan-Lee [4] showed that it is possible to take $N=6$ in Higman's result. The largest finite 2-generator group of exponent 5 has class 12 ([5]), and the largest finite 3-generator group of exponent 5 has class 17 ([15]). So $N=6$ is best possible, at least for small values of $m$. In this article we show that if $G$ is a finite $m$-generator group of exponent 7 then $G$ is nilpotent of class at most $51 \mathrm{~m}^{8}$. Although this result is unlikely to be best possible, it is still considerably better than the bound

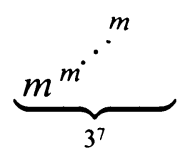

mentioned above.

The results of Higman, Kostrikin, and Zelmanov all make use of the connection between groups of prime-power exponent and their associated Lie rings. If $G$ is a finite $m$-generator group of exponent $p$, and if $L$ is the associated Lie ring of $G$, then $L$ is an $m$-generator Lie ring of characteristic $p$, and $L$ satisfies the Engel identity $x y^{p-1}=0$. (See [16] for a proof of this fact.) We can think of $L$ as a Lie algebra over the field of integers modulo $p$, and Kostrikin solved the restricted Burnside problem for exponent $p$ by proving that if $L$ is a finitely generated Lie algebra over a field of characteristic $p$, and if $L$ satisfies the Engel- $(p-1)$ identity, then $L$ is nilpotent (of bounded class). Since the nilpotency class of a finite group of exponent $p$ is the same as the nilpotency class of its associated Lie ring, his theorem proves that the class of a finite $m$-generator group of exponent $p$ is bounded.

The connection with the restricted Burnside problem has motivated a number of researchers to investigate Engel Lie algebras. It is well known that if $L$ is an Engel-2 Lie algebra over a field $K$, then $L$ is nilpotent of class at most 3 . (For a proof see [16].) If $L$ is an Engel-3 Lie algebra over a field $K$, then $L$ is nilpotent of class at most 4 provided $\operatorname{char}(K) \neq 2,5$ (see [6] and [13]). In the case when $\operatorname{char}(K)=2$, then an $m$-generator Engel-3 Lie algebra over $K$ is nilpotent of class at most $2(m+1)^{6}$ (see [14]), and in the case when $\operatorname{char}(K)=5$, then an $m$-generator Engel-3 Lie algebra over $K$ is nilpotent of 
class at most $2 m$. Engel- 4 Lie algebras are nilpotent of class at most 8 provided the characteristic of the ground field is not 2, 3, or 5 (see [13]). Traustason [14] has shown that an $m$-generator Engel-4 Lie algebra over a field of characteristic 2 is nilpotent of class at most $2(m+1)^{6}$. Over fields of characteristic 3 and 5 , the class of an $m$-generator Engel-4 Lie algebra is bounded by $3 m$ and $6 m$ respectively. It is easy to see that if $L$ is an Engel-p Lie algebra over a field of characteristic $p$, then $a b^{p-1}$ is central in $L$ for all $a, b \in L$. So if we let $\zeta(L)$ be the centre of $L$, then $L / \zeta(L)$ is Engel- $(p-1)$. It follows from this and from the results on Engel-4 Lie algebras, that the class of an $m$-generator Engel-5 Lie algebra over a field of characteristic 5 is at most $6 m+1$. Traustason [14] has shown that if $\operatorname{char}(K)>5$, then an $m$-generator Engel-5 Lie algebra over $K$ is nilpotent of class at most $79 \mathrm{~m}$.

A number of results mentioned above make use of a reduction due to Higman [7]. He introduced a Lie algebra $L$ defined as follows. We let $F$ be the free Lie algebra with free generators $x, a_{1}, a_{2}, \ldots$ over a field $K$. If we let $I$ be the ideal of $F$ generated by $\left\{a b^{n} \mid a, b \in F\right\}$, then $F / I$ is a (relatively) free Lie algebra in the variety of Lie algebras determined by the Engel- $n$ identity $x y^{n}=0$. We let $L=F /(I+J)$, where $J$ is the ideal of $F$ generated by $\left\{a_{i} a_{j} \mid i, j \geq 1\right\}$. So if we set $\bar{x}=x+I+J$, and $\bar{a}_{i}=a_{i}+I+J$ for $i=1,2, \ldots$, then $L$ is an Engel-n Lie algebra over $K, L$ is generated by $\bar{x}, \bar{a}_{1}, \bar{a}_{2}, \ldots$, and $\bar{a}_{i} \bar{a}_{j}=0$ for all $i, j \geq 1$. It is easy to see that provided $\operatorname{char}(K)>n$, then $L$ is nilpotent if and only if the ideal of $L$ generated by $\bar{x}$ is nilpotent, and Higman showed that if this ideal is nilpotent of class $N$ then the ideal of $F / I$ generated by $x+I$ is also nilpotent of class $N$. So, if $I d_{L}(\bar{x})$ is nilpotent of class $N$, it follows that if $x$ is an arbitrary element of an arbitrary Engel- $n$ Lie algebra $M$ over $K$, then the ideal of $M$ generated by $x$ is nilpotent of class at most $N$. This implies that $m$-generator Engel$n$ Lie algebras over $K$ are nilpotent of class at most $N m$. Higman showed that $L$ is nilpotent in the case when $n=4$, provided that $\operatorname{char}(K) \neq 2,3$. Higman did not explicitly compute the class of $L$, but Havas, Newman, and Vaughan-Lee [4] used a computer program to compute $L$ when $n=4$ and $\operatorname{char}(K)=5$. They found that $L$ has class 12 in this case, and that the ideal of $L$ generated by $\bar{x}$ is nilpotent of class 6 . It is from this result that we deduce that if $G$ is a finite $m$-generator group of exponent 5 then $G$ has class at most $6 \mathrm{~m}$. Higman's algebra $L$ is also known to be nilpotent for the case $n=3$, $\operatorname{char}(K) \neq 2$, and for the case $n=5, \operatorname{char}(K) \neq 2,3$ (see [14]). On the other hand Traustason [13] has found Engel-3 Lie algebras of characteristic 2 in which the ideal generated by an element is not nilpotent, and I have similar examples of Engel-5 Lie algebras of characteristic 3.

In this article we consider a certain quotient algebra $M$ of Higman's algebra $L$. We let $X$ be the ideal of $F$ generated by $\left\{x a_{i} a_{j} \mid i, j \geq 1\right\}$, and we let $M=F /(I+J+X)$. We show that if $\operatorname{char}(K)>n$ then the nilpotency of $M$ implies that $I d_{A}\left(a b^{n-2}\right)$ is nilpotent whenever $a, b$ are elements of an Engel$n$ Lie algebra $A$ over $K$. I have used an implementation of the nilpotent quotient algorithm for graded Lie rings (see [4]) to compute $M$ for $n=6$ and $\operatorname{char}(K)=7$. The computations show that $M$ is nilpotent of class 22 in this case. We are able to deduce from this, and from other properties of $M$, that $m$-generator Engel-6 Lie algebras of characteristic 7 are nilpotent of class at 
most $51 \mathrm{~m}^{8}$. It follows from this that finite $m$-generator groups of exponent 7 are nilpotent of class at most $51 \mathrm{~m}^{8}$.

\section{REDUCTION THEOREMS}

As above, we let $K$ be an arbitrary field, and we let $F$ be the free Lie algebra over $K$ with free generators $x, a_{1}, a_{2}, \ldots$ If $u$ is any (Lie) product of these free generators then we define the multiweight of $u$ to be $\underline{w}=$ $\left(w_{0}, w_{1}, w_{2}, \ldots\right)$ where $w_{0}$ is the number of times $x$ appears in the product $u$, and where for each $i=1,2, \ldots, w_{i}$ is the number of times the free generator $a_{i}$ occurs in the product. Note that $w_{i}=0$ for all but finitely many values of $i$. If $\underline{w}$ is any given multiweight then we let $F_{\underline{w}}$ be the $K$-subspace of $F$ spanned by products of multiweight $\underline{w}$. We call $F_{w}$ the multihomogeneous component of $F$ of multiweight $\underline{w}$. The free Lie algebra $F$ is multigraded in the sense that it is the direct sum of its multihomogeneous components, and satisfies $F_{\underline{v}} F_{\underline{w}} \leq F_{\underline{v}} \underline{w}$ for all multiweights $\underline{v}$ and $\underline{w}$. (Here addition of multiweights is defined componentwise.) We suppose that $\operatorname{char}(K)>n$, which implies that the Engel- $n$ identity $x y^{n}=0$ is equivalent to the multilinear identity $S_{n}\left(x, y_{1}, y_{2}, \ldots, y_{n}\right)=0$, where

$$
S_{n}\left(x, y_{1}, y_{2}, \ldots, y_{n}\right)=\sum_{\sigma \in \operatorname{Sym}(n)} x y_{\sigma(1)} y_{\sigma(2)} \cdots y_{\sigma(n)} .
$$

To see this, substitute $y_{1}+y_{2}+\cdots+y_{n}$ for $y$ in $x y^{n}$, expand, and pick out the terms which are multilinear in $y_{1}, y_{2}, \ldots, y_{n}$. We obtain the multilinear word $S_{n}\left(x, y_{1}, y_{2}, \ldots, y_{n}\right)$. On the other hand if we substitute $y$ for $y_{1}, y_{2}, \ldots, y_{n}$ in $S_{n}\left(x, y_{1}, y_{2}, \ldots, y_{n}\right)$ then we obtain $n ! x y^{n}$. So the ideal $I$ of $F$ generated by values of $x y^{n}$ is generated by values of $S_{n}\left(x, y_{1}, y_{2}, \ldots, y_{n}\right)$. It follows that $I$ is a multigraded ideal of $F$, and hence that the relatively free Engel- $n$ Lie algebra $F / I$ is multigraded. The ideal $J$ of $F$ generated by $\left\{a_{i} a_{j} \mid i, j \geq 1\right\}$ is also multigraded. As above we let $L=F /(I+J)$ and we let $\bar{x}=x+I+J, \bar{a}_{i}=a_{i}+I+J$ for $i=1,2, \ldots$. The following lemma was mentioned above, and is due to Higman [7].

Lemma 1. Suppose that $I d_{L}(\bar{x})$ is nilpotent of class $N$. If $u$ is a product of the free generators of $F$, and if $u$ has multiweight $\left(w_{0}, w_{1}, w_{2}, \ldots\right)$ where $w_{0}>N$, then $u \in I$.

Proof. The proof is by induction on $\sum_{i>0} w_{i}$. First note that the result is trivial if $\sum_{i>0} w_{i}=0$. Now let $u$ have multiweight $\left(w_{0}, w_{1}, w_{2}, \ldots\right)$ where $w_{0}>N$ and where $\sum_{i>0} w_{i}>0$. Then

$$
u+I+J \in\left(I_{L}(\bar{x})\right)^{w_{0}}=\{0\},
$$

and so $u \in I+J$. Since $F, I$, and $J$ are all multigraded this means that we can express $u$ in the form $u=a+b$, where $a \in I$ and $b \in J$, and where $a$ and $b$ have the same multiweight as $u$. Since $b \in J$ we can express $b$ as a linear combination of products $c$ of the form $c=a_{i} a_{j} v_{1} v_{2} \cdots v_{m}$ where $v_{1}, v_{2}, \ldots, v_{m}$ are elements from the generating set $\left\{x, a_{1}, a_{2}, \ldots\right\}$ of $F$, and where $c$ has multiweight $\left(w_{0}, w_{1}, w_{2}, \ldots\right)$. We let $c$ be a product of this form, and we pick an index $k$ such that $w_{k}=0$. Then we let $d=a_{k} v_{1} v_{2} \cdots v_{m}$. Clearly $d$ has multiweight $\left(w_{0}, w_{1}, \ldots, w_{i}-1, \ldots, w_{j}-1, \ldots, w_{k}+1, \ldots\right)$ and so by induction $d \in I$. Now consider the endomorphism of the free Lie algebra $F$ 
which maps $x$ to $x$, maps $a_{r}$ to $a_{r}$ for $r \neq k$, and maps $a_{k}$ to $a_{i} a_{j}$. The product $c$ is the image of $d$ under this endomorphism, and so since $d \in I$ and since $I$ is fully invariant, we see that $c \in I$. Since $b$ is a linear combination of products $c$ of this form, this implies that $b \in I$. Hence $u \in I$, as required.

In this article we make use of the following variation on Lemma 1 . We let $F, I$, and $J$ be as above, and we let $X$ be the ideal of $F$ generated by $\left\{x a_{i} a_{j} \mid i, j \geq 1\right\}$. Then we let $M=F /(I+J+X)$, and let $\tilde{x}=x+I+J+X$, $\tilde{a}_{i}=a_{i}+I+J+X$ for $i=1,2, \ldots$.

Lemma 2. Suppose that $I d_{M}(\tilde{x})$ is nilpotent of class $N$. If $u$ is a product of the free generators of $F$, and if $u$ has multiweight $\left(w_{0}, w_{1}, w_{2}, \ldots\right)$ where $w_{0}-\sum_{i>0} w_{i}>N$, then $u \in I$.

Proof. The proof is by induction on $\sum_{i>0} w_{i}$. First note that the result is trivial if $\sum_{i>0} w_{i}=0$. Now let $u$ have multiweight $\left(w_{0}, w_{1}, w_{2}, \ldots\right)$ where $w_{0}-\sum_{i>0} w_{i}>N$ and where $\sum_{i>0} w_{i}>0$. Then

$$
u+I+J+X \in\left(\operatorname{Id}_{L}(\tilde{x})\right)^{w_{0}} \leq\left(\operatorname{Id}_{L}(\tilde{x})\right)^{N+1}=\{0\},
$$

and so $u \in I+J+X$. Since $F, I, J$, and $X$ are all multigraded this means that we can express $u$ in the form $u=a+b+c$, where $a \in I, b \in J$, and $c \in X$, and where $a, b$, and $c$ have the same multiweight as $u$. The proof that $b \in I$ is exactly the same as in the proof of Lemma 1, and the proof that $c \in I$ is very similar. Consider an element $c \in X$ with multiweight $\left(w_{0}, w_{1}, w_{2}, \ldots\right)$. We can express $c$ as a linear combination of products $d$ of the form $d=\left(x a_{i} a_{j}\right) v_{1} v_{2} \ldots v_{m}$ where $v_{1}, v_{2}, \ldots, v_{m}$ are elements from the generating set $\left\{x, a_{1}, a_{2}, \ldots\right\}$ of $F$, and where $d$ has multiweight $\left(w_{0}, w_{1}, w_{2}, \ldots\right)$. We let $d$ be a product of this form, and we pick an index $k$ such that $w_{k}=0$. Then we let $e=a_{k} v_{1} v_{2} \cdots v_{m}$. Clearly $e$ has multiweight $\left(w_{0}-1, w_{1}, \ldots, w_{i}-1, \ldots, w_{j}-1, \ldots, w_{k}+1, \ldots\right)$ and so by induction $e \in I$. Now consider the endomorphism of the free Lie algebra $F$ which maps $x$ to $x$, maps $a_{r}$ to $a_{r}$ for $r \neq k$, and maps $a_{k}$ to $x a_{i} a_{j}$. The product $d$ is the image of $e$ under this endomorphism, and so since $e \in I$ and since $I$ is fully invariant, we see that $d \in I$. Since $c$ is a linear combination of products $d$ of this form, we see that $c \in I$. We have already noted that $b \in I$, and so $u \in I$, as required.

We use Lemma 2 to establish the following reduction theorem.

Theorem 3. Suppose that $I d_{M}(\tilde{x})$ is nilpotent of class $N$, and let $A$ be an arbitrary Engel-n Lie algebra over the field $K$. If $a$ and $b$ are arbitrary elements of $A$ then the ideal of $A$ generated by $a b^{n-2}$ is nilpotent of class at most $8(N-n)+41$.

Proof. The basic idea of the proof is very simple, although it relies on detailed calculations from $\S \S 3,4$, and 5. If $x$ is any element of $A$ and if $m>2$, then it follows from Lemma 6 in $\S 5$ that $\left(I d_{A}(x)\right)^{m}$ is contained in the ideal of $A$ generated by products of the form

$$
v=x a_{11} a_{12} \cdots a_{1 k_{1}} x a_{21} a_{22} \cdots a_{2 k_{2}} x \cdots x a_{r 1} a_{r 2} \cdots a_{r k_{r}},
$$

where $v$ has weight $r$ in $x$ with $\frac{3 m+3}{8} \leq r \leq m$, and where $v$ has total wieght $r+k_{1}+k_{2}+\cdots+k_{r}<r+2+\frac{(r-1)(3 n-10)}{3}$. If we let $x=a b^{n-2}$ then this 
implies that $\left(\operatorname{Id}_{A}(x)\right)^{m}$ is contained in the ideal of $A$ generated by products which have weight $r(n-2)$ in $b$ for some $r$ such that $\frac{3 m+3}{8} \leq r \leq m$ and have total weight less than $r(n-1)+2+\frac{(r-1)(3 n-10)}{3}$. Lemma 2 implies that these products are zero provided $r(n-2)-\left(r+2+\frac{(r-1)(3 n-10)}{3}\right) \geq N$, that is, if $r \geq 3(N-n)+16$, or if $m \geq 8(N-n)+42$.

Note that the conclusions of Lemma 2 and Theorem 3 are still valid (with the same proofs) under a slightly weaker hypothesis. We can replace the hypothesis that $\operatorname{Id}_{M}(\tilde{x})$ is nilpotent of class $N$ with the hypothesis that if $v$ is a product in $M$ with multiweight $\left(w_{0}, w_{1}, w_{2}, \ldots\right)$ in the generators $\tilde{x}, \tilde{a}_{1}, \tilde{a}_{2}, \ldots$, then $v=0$ whenever $w_{0}-\sum_{i>0} w_{i}>N$.

\section{ENGEL- $(p-1)$ LiE ALGEBRAS}

Let $L$ be a Lie algebra over a field $K$ of characteristic $p>5$, and let $L$ satisfy the Engel- $(p-1)$ identity. We suppose that $L$ has an abelian subalgebra $A$, and an element $x$ such that $L$ is generated by $A$ and $x$. The Engel$(p-1)$ identity implies that $u v^{p-1}=0$ for all $u, v \in L$. If we substitute $v_{1}+v_{2}+\cdots+v_{p-1}$ for $v$, expand, and pick out the terms which are linear in $v_{1}, v_{2}, \ldots, v_{p-1}$, then we obtain the identity

$$
S_{p-1}\left(u, v_{1}, v_{2}, \ldots, v_{p-1}\right)=0,
$$

where

$$
S_{p-1}\left(u, v_{1}, v_{2}, \ldots, v_{p-1}\right)=\sum_{\sigma \in \operatorname{Sym}(p-1)} u v_{\sigma(1)} v_{\sigma(2)} \cdots v_{\sigma(p-1)} .
$$

We will use this identity to establish a number of further identities that hold in $L$. In the identities that follow we let $a, b, c, a_{1}, a_{2}, \ldots, b_{1}, b_{2}, \ldots$, $c_{1}, c_{2}, \ldots$ denote arbitrary elements of the abelian Lie subalgebra $A$ of $L$. We state each identity in turn, and follow each statement with a proof.

$$
u a_{1} a_{2} \cdots a_{p-1}=0 \text { for all } u \in L .
$$

This follows immediately from the identity $S_{p-1}\left(u, a_{1}, a_{2}, \ldots, a_{p-1}\right)=0$, using the fact that $a_{i} a_{j}=0$ for $1 \leq i, j \leq p-1$.

$$
x a^{p-3} x b^{p-2}=x a^{p-2} x b^{p-3}=0 .
$$

Using the fact that $p-3$ is even, we write $p-3=2 s$. Then

$$
0=\left(x a^{s}\right)\left(x a^{s}\right)=\sum_{r=0}^{s}(-1)^{r}\left(\begin{array}{l}
s \\
r
\end{array}\right) x a^{s+r} x a^{s-r},
$$

and so

$$
x a^{p-3} x b^{p-2}= \pm \sum_{r=0}^{s-1}(-1)^{r}\left(\begin{array}{l}
s \\
r
\end{array}\right) x a^{s+r} x a^{s-r} b^{p-2},
$$

which is zero by (1). So $x a^{p-3} x b^{p-2}=0$. Substituting $b_{1}+b_{2}+\cdots+b_{p-2}$ for $b$, expanding, and picking out the terms which are linear in $b_{1}, b_{2}, \ldots, b_{p-2}$, we obtain $x a^{p-3} x b_{1} b_{2} \cdots b_{p-2}=0$. (We refer to this as linearizing with respect 
to $b$, and we will be making frequent use of this process.) Next consider $x a^{p-2} x b^{p-3}$. The identity $S_{p-1}(x, x, a, a, \ldots, a)=0$ gives

$$
x a^{p-2} x=-\sum_{r=0}^{p-2} x a^{r} x a^{p-2-r},
$$

and so

$$
x a^{p-2} x b^{p-3}=-\sum_{r=0}^{p-3} x a^{r} x a^{p-2-r} b^{p-3}=0,
$$

by (1), and by the identity $x a^{p-3} x b_{1} b_{2} \cdots b_{p-2}=0$ just established.

Now we let $Y$ be the ideal of $L$ generated by all elements of the form $x a_{1} a_{2} \cdots a_{p-2}$, with $a_{1}, a_{2}, \ldots, a_{p-2} \in A$. In the remainder of this section we will establish a number of identities which hold in the quotient algebra $L / Y$.

$$
x a^{p-4} x b^{p-2} \in Y \text {. }
$$

The identity $S_{p-1}\left(x a^{p-4} x, b, b, \ldots, b\right)=0$ gives

$$
\sum_{r=0}^{p-2} x a^{p-4} b^{r} x b^{p-2-r}=0,
$$

and using the fact that $x a^{p-4}\left(x b^{p-2}\right) \in Y$ we obtain

$$
\sum_{r=0}^{p-2}(-1)^{r}\left(\begin{array}{c}
p-2 \\
r
\end{array}\right) x a^{p-4} b^{r} x b^{p-2-r} \in Y .
$$

Now $x a^{p-4} b^{r} \in Y$ if $r>1$, and so these two identities give

$$
\begin{gathered}
x a^{p-4} x b^{p-2}+x a^{p-4} b x b^{p-3} \in Y, \\
x a^{p-4} x b^{p-2}-(p-2) x a^{p-4} b x b^{p-3} \in Y .
\end{gathered}
$$

Identity (3) follows immediately.

$$
x a^{p-3} x b^{p-3} \in Y .
$$

Using the same argument as in the proof of (2) we see that if we let $s=$ $(p-3) / 2$ then

$$
x a^{p-3} x b^{p-3}= \pm \sum_{r=0}^{s-1}(-1)^{r}\left(\begin{array}{l}
s \\
r
\end{array}\right) x a^{s+r} x a^{s-r} b^{p-3} .
$$

Using (1) this gives

$$
x a^{p-3} x b^{p-3}= \pm(-1)^{s-1} s x a^{p-4} x a b^{p-3},
$$

and so (4) follows from the linearization of (3) with respect to $b$.

In the remainder of this section we will establish a number of identities involving elements of the form

$$
x a_{1} a_{2} \cdots a_{r} x b_{1} b_{2} \cdots b_{s} x c_{1} c_{2} \cdots c_{t},
$$


where $a_{1}, a_{2}, \ldots, a_{r}, b_{1}, b_{2}, \ldots, b_{s}, c_{1}, c_{2}, \ldots, c_{t} \in A$, and where $r+s+t=$ $3 p-11$. We let $M$ be the subspace of $L$ spanned by elements of this form. Our aim is to prove that all these products lie in $Y$, so that $M \leq Y$. Clearly such a product lies in $Y$ if $r \geq p-2$. Identity (1) implies that the product is zero if $s \geq p-1$, or $t \geq p-1$. Identity (2) implies that the product is zero if $r+s \geq 2 p-5$, and identities (3) and (4) imply that the product lies in $Y$ if $r+s=2 p-6$. So we need only consider products with $r \leq p-3$, and with $2 p-9 \leq r+s \leq 2 p-7$.

$$
x a^{r} x b^{s} x c^{t} \in Y \quad \text { if } r+s+t=3 p-11, r \leq p-5 .
$$

Note that we may assume that $r<p-2$, and that $s, t<p-1$, and so the linearization of (5) gives

$$
x a_{1} a_{2} \cdots a_{r} x b_{1} b_{2} \cdots b_{s} x c_{1} c_{2} \cdots c_{t} \in Y
$$

if $r+s+t=3 p-11, r \leq p-5$.

First we deal with the case when $r \leq p-6$. In this case either $s=p-2$ or $t=p-2$. Suppose that $t=p-2$. Then $S_{p-1}\left(x a^{r} x b^{s}, x, c, c, \ldots, c\right)=0$ gives

$$
\sum_{m=0}^{p-2} x a^{r} x b^{s} c^{m} x c^{p-2-m}=0 .
$$

Also, using the fact that $x a^{r} x b^{s}\left(x c^{p-2}\right) \in Y$, we obtain

$$
\sum_{m=0}^{p-2}(-1)^{m}\left(\begin{array}{c}
p-2 \\
m
\end{array}\right) x a^{r} x b^{s} c^{m} x c^{p-2-m} \in Y .
$$

Now if $r \leq p-6, r+s+t=3 p-11, t=p-2$, then $s \geq p-3$. So if $m>1$ then $x a^{r} x b^{s} c^{m}=0$ by (1). So the two identities above give

$$
\begin{gathered}
x a^{r} x b^{s} x c^{p-2}+x a^{r} x b^{s} c x c^{p-3}=0, \\
x a^{r} x b^{s} x c^{p-2}-(p-2) x a^{r} x b^{s} c x c^{p-3} \in Y .
\end{gathered}
$$

The fact that $x a^{r} x b^{s} x c^{p-2} \in Y$ (when $r \leq p-6, r+s=2 p-9$ ) follows immediately from this. The case when $s=p-2$ follows similarly from the identity $S_{p-1}\left(x a^{r} x, x, b, b, \ldots, b\right) c^{t}=0$, and from the fact that $x a^{r} x\left(x b^{p-2}\right) c^{t} \in Y$.

Now consider the case when $r=p-5$. Just as in the proof of (2), we use the fact that $p-5$ is even, and we let $s=(p-5) / 2$. Then

$$
0=\left(x a^{s}\right)\left(x a^{s}\right)=\sum_{r=0}^{s}(-1)^{r}\left(\begin{array}{l}
s \\
r
\end{array}\right) x a^{s+r} x a^{s-r},
$$

and so

$$
x a^{p-5} x= \pm \sum_{r=0}^{s-1}(-1)^{r}\left(\begin{array}{l}
s \\
r
\end{array}\right) x a^{s+r} x a^{s-r} .
$$

So the case when $r=p-5$ reduces to the case when $r \leq p-6$, and the proof of $(5)$ is complete.

$$
x a^{r} x a^{p-2-r} b^{s} x c^{t} \in Y \quad \text { if } 0 \leq r \leq p-2, s+t=2 p-9 .
$$


Identity (5) implies (6) if $r \leq p-5$, and clearly (6) holds if $r=p-2$. So we need only consider the cases when $r=p-4$ or $p-3$. The identity $S_{p-1}(x, x, a, a, \ldots, a) b^{s} x c^{t}=0$ gives

$$
x a^{p-4} x a^{2} b^{s} x c^{t}+x a^{p-3} x a b^{s} x c^{t} \in Y,
$$

and the fact that $x\left(x a^{p-2}\right) b^{s} x c^{t} \in Y$ gives

$$
-\left(\begin{array}{c}
p-2 \\
2
\end{array}\right) x a^{p-4} x a^{2} b^{s} x c^{t}+(p-2) x a^{p-3} x a b^{s} x c^{t} \in Y .
$$

This completes the proof of $(6)$.

$$
x a^{p-3} x b^{p-6} c^{r} x c^{p-2-r} \in Y \text { for } 0 \leq r \leq p-2 .
$$

Identities (1)-(4) imply (7) for $r \geq 3$. The identity

$$
S_{p-1}\left(x a^{p-3}, x b^{p-6} x, c, c, \ldots, c\right)=0
$$

gives $x a^{p-3}\left(x b^{p-6} x\right) c^{p-2} \in Y$. If we expand this and use (1) then we obtain

$$
2 x a^{p-3} x b^{p-6} x c^{p-2} \in Y,
$$

which gives (7) for the case $r=0$. Using these results we see that the identity $S_{p-1}\left(x a^{p-3} x b^{p-6}, x, c, c, \ldots, c\right)=0$ gives

$$
x a^{p-3} x b^{p-6} c x c^{p-3}+x a^{p-3} x b^{p-6} c^{2} x c^{p-4} \in Y,
$$

and the fact that $x a^{p-3} x b^{p-6}\left(x c^{p-2}\right) \in Y$ implies that

$$
-(p-2) x a^{p-3} x b^{p-6} c x c^{p-3}+\left(\begin{array}{c}
p-2 \\
2
\end{array}\right) x a^{p-3} x b^{p-6} c^{2} x c^{p-4} \in Y .
$$

This completes the proof of (7).

$$
x a_{1} a_{2} \cdots a_{r} b^{s} x b^{p-2-s} c_{1} c_{2} \cdots c_{t} x d_{1} d_{2} \cdots d_{u} \in Y
$$

if $0 \leq s \leq p-2, r+t+u=2 p-9$.

We prove (8) by induction on $r$. The case $r=0$ follows from (6), so suppose that $r>0$ and suppose that (8) holds for all smaller values of $r$. Clearly (8) holds if $r+s \geq p-2$, so we suppose that $r+s<p-2$. We let $u=a_{1}+a_{2}+\cdots+a_{r}+b$. Then (6) implies that

$$
x u^{r+s} x u^{p-2-r-s} b^{r} c_{1} c_{2} \cdots c_{t} x d_{1} d_{2} \cdots d_{u} \in Y .
$$

If we expand this identity and pick out the terms which are linear in $a_{1}$, $a_{2}, \ldots, a_{r}$ and have degree $p-2$ in $b$, then using the inductive hypothesis we obtain

$$
r !\left(\begin{array}{c}
r+s \\
s
\end{array}\right) x a_{1} a_{2} \cdots a_{r} b^{s} x b^{p-2-s} c_{1} c_{2} \cdots c_{t} x d_{1} d_{2} \cdots d_{u} \in Y .
$$

This establishes (8).

$$
x a^{p-3} x b_{1} b_{2} \cdots b_{r} c^{s} x c^{p-2-s} d_{1} d_{2} \cdots d_{t} \in Y
$$

if $0 \leq s \leq p-2, r+t=p-6$. 
We establish (9) by induction on $t$, using (7), in the same way as we used (6) to establish (8).

(10) $x a^{p-3} x b^{p-5} x c^{p-3}=\alpha x a^{p-3} x b^{p-5} c x c^{p-4}$ modulo $Y$, for some $\alpha \neq 0$.

If we use (4) and (7) then the identity $S_{p-1}\left(x a^{p-3}, x, x, c, c, \ldots, c\right) b^{p-5}=$ 0 gives

$$
x a^{p-3} x c^{p-5} x c^{2} b^{p-5}+x a^{p-3} x c^{p-4} x c b^{p-5} \in Y .
$$

We let $u=b+c$, and use (9), which gives

$$
x a^{p-3} x u^{p-5} x u^{3} b^{p-6} \in Y .
$$

We expand this identity, and pick out the terms which are of degree $p-5$ in $b$ and degree $p-3$ in $c$. This gives

$$
3 x a^{p-3} x c^{p-5} x c^{2} b^{p-5}+(p-5) x a^{p-3} x b c^{p-6} x c^{3} b^{p-6} \in Y .
$$

Using (9) again we have $x a^{p-3} x b u^{p-6} x u^{4} b^{p-7} \in Y$. Expanding this we obtain

$$
4 x a^{p-3} x b c^{p-6} x c^{3} b^{p-6}+(p-6) x a^{p-3} x b^{2} c^{p-7} x c^{4} b^{p-7} \in Y \text {. }
$$

So we can see that $x a^{p-3} x c^{p-5} x c^{2} b^{p-5}$ is a nonzero linear multiple of $x a^{p-3} x b^{2} c^{p-7} x c^{4} b^{p-7}$. Continuing in this way we see that $x a^{p-3} x c^{p-5} x c^{2} b^{p-5}$ is a nonzero linear multiple of $x a^{p-3} x b^{p-5} x c^{p-3}$. Similarly we see that $x a^{p-3} x c^{p-4} x c b^{p-5}$ is a nonzero linear multiple of $x a^{p-3} x b^{p-5} c x c^{p-4}$. So (10) follows from the identity $x a^{p-3} x c^{p-5} x c^{2} b^{p-5}+x a^{p-3} x c^{p-4} x c b^{p-5} \in Y$ proved above.

$$
x a^{p-4} x b^{p-6} c^{r} x c^{p-1-r} \in Y \text { for } 0 \leq r \leq p-1 .
$$

It is clear that $x a^{p-4} x b^{p-6} c^{r} x c^{p-1-r}$ must lie in the ideal of $L$ generated by elements of the form $x a_{1} a_{2} \cdots a_{p-3}$. So, modulo $Y, x a^{p-4} x b^{p-6} c^{r} x c^{p-1-r}$ can be expressed as a linear combination of elements of the form

$$
x a_{1} a_{2} \cdots a_{p-3} x b_{1} b_{2} \cdots b_{r} x c_{1} c_{2} \cdots c_{s}
$$

where $r+s=2 p-8$. Using (2), (4), (7), and (10) we may assume that $r=p-4$. So, modulo $Y, x a^{p-4} x b^{p-6} c^{r} x c^{p-1-r}$ can be expressed as a linear combination of terms of the form

$$
x c^{r} a_{1} a_{2} \cdots a_{p-3-r} x c^{s} b_{1} b_{2} \cdots b_{p-4-s} x c^{t} d_{1} d_{2} \cdots d_{p-4-t}
$$

where $r+s+t=p-1$. We prove that these elements all lie in $Y$ by induction on $t$. If $t<2$ then elements of this form lie in $Y$ by (8). So suppose that $t \geq 2$. Let $u=b_{1}+b_{2}+\cdots+b_{p-4-s}+c$. Then (9) gives

$$
x c^{r} a_{1} a_{2} \cdots a_{p-3-r} x u^{p-4} x u^{2} c^{t-2} d_{1} d_{2} \cdots d_{p-4-t} \in Y .
$$

Expanding this and picking out the terms which are linear in $b_{1}, b_{2}, \ldots, b_{p-4-s}$ and of degree $p-1$ in $c$ we see that

$$
x c^{r} a_{1} a_{2} \cdots a_{p-3-r} x c^{s} b_{1} b_{2} \cdots b_{p-4-s} x c^{t} d_{1} d_{2} \cdots d_{p-4-t}
$$

is a linear combination of terms which have the same form, but have smaller values of $t$. So all these elements lie in $Y$ by induction on $t$. This establishes (11).

$$
x a^{p-4} x b^{p-5} c^{r} x c^{p-2-r} \in Y \quad \text { for } 0 \leq r \leq p-2 .
$$


Using (1) and (3) we see that $S_{p-1}\left(x a^{p-4} x b^{p-5}, x, c, c, \ldots, c\right)=0$ gives

$$
x a^{p-4} x b^{p-5} x c^{p-2}+x a^{p-4} x b^{p-5} c x c^{p-3}+x a^{p-4} x b^{p-5} c^{2} x c^{p-4} \in Y .
$$

Also the fact that $x a^{p-4} x b^{p-5}\left(x c^{p-2}\right) \in Y$ gives

$$
x a^{p-4} x b^{p-5} x c^{p-2}+2 x a^{p-4} x b^{p-5} c x c^{p-3}+3 x a^{p-4} x b^{p-5} c^{2} x c^{p-4} \in Y .
$$

Finally, $S_{p-1}\left(x a^{p-4} x b^{p-6}, x, c, c, \ldots, c\right) b=0$ together with (1) gives

(15) $x a^{p-4} x b^{p-6} c x c^{p-3} b+x a^{p-4} x b^{p-6} c^{2} x c^{p-4} b+x a^{p-4} x b^{p-6} c^{3} x c^{p-5} b \in Y$.

If we set $u=b+c$, then (11) gives $x a^{p-4} x b^{p-6} u x u^{p-2} \in Y$. Expanding this, and picking out the terms of degree $p-5$ in $b$ and of degree $p-2$ in $c$ then we obtain

$$
(p-2) x a^{p-4} x b^{p-6} c x c^{p-3} b+x a^{p-4} x b^{p-5} x c^{p-2} \in Y .
$$

Similarly,

$$
(p-3) x a^{p-4} x b^{p-6} c^{2} x c^{p-4} b+2 x a^{p-4} x b^{p-5} c x c^{p-3} \in Y,
$$

and

$$
(p-4) x a^{p-4} x b^{p-6} c^{3} x c^{p-5} b+3 x a^{p-4} x b^{p-5} c^{2} x c^{p-4} \in Y .
$$

So $(15)$ gives

$$
\begin{aligned}
& \frac{1}{p-2} x a^{p-4} x b^{p-5} x c^{p-2}+\frac{2}{p-3} x a^{p-4} x b^{p-5} c x c^{p-3} \\
& +\frac{3}{p-4} x a^{p-4} x b^{p-5} c^{2} x c^{p-4} \in Y .
\end{aligned}
$$

This, together with (13) and (14) gives (12).

$$
x a^{p-3} x b^{p-5} x c^{p-3} \in Y .
$$

Using (5), (7), and (12) we see that $S_{p-1}\left(x b^{p-5}, x, x, a, a, \ldots, a\right) c^{p-3}=0$ gives

$$
x b^{p-5} a x a^{p-4} x c^{p-3}+x b^{p-5} a^{2} x a^{p-5} x c^{p-3} \in Y .
$$

If we set $u=a+b$ then (8) gives $x b^{p-6} u^{2} x u^{p-4} x c^{p-3} \in Y$. Expanding, and picking out the terms of degree $p-3$ in $a$ and degree $p-5$ in $b$ we obtain

$$
2 x b^{p-5} a x a^{p-4} x c^{p-3}+(p-4) x b^{p-6} a^{2} x a^{p-5} b x c^{p-3} \in Y .
$$

Similarly $x b^{p-4-r} u^{r} x u^{p-2-r} b^{r-2} x c^{p-3} \in Y$ gives

$$
r x b^{p-3-r} a^{r-1} x a^{p-2-r} b^{r-2} x c^{p-3}+(p-2-r) x b^{p-4-r} a^{r} x a^{p-3-r} b^{r-1} x c^{p-3} \in Y
$$

for $r=3,4, \ldots, p-4$. Hence

$$
x b^{p-5} a x a^{p-4} x c^{p-3}-x a^{p-4} x a b^{p-5} x c^{p-3} \in Y .
$$

Similarly

$$
x b^{p-5} a^{2} x a^{p-5} x c^{p-3}-\frac{2}{(p-4)(p-3)} x a^{p-3} x b^{p-5} x c^{p-3} \in Y,
$$

and so (17) gives

$$
x a^{p-4} x a b^{p-5} x c^{p-3}+\frac{2}{(p-4)(p-3)} x a^{p-3} x b^{p-5} x c^{p-3} \in Y .
$$


Now, as we saw in the proof of (2), if we let $s=(p-3) / 2$ then

$$
0=\left(x a^{s}\right)\left(x a^{s}\right)=\sum_{r=0}^{s}(-1)^{r}\left(\begin{array}{l}
s \\
r
\end{array}\right) x a^{s+r} x a^{s-r} .
$$

It follows that

$$
(-1)^{s-1} s x a^{p-4} x a b^{p-5} x c^{p-3}+(-1)^{s} x a^{p-3} x b^{p-5} x c^{p-3} \in Y,
$$

and this, together with the identity above, gives (16).

$$
x a^{p-3} x b^{r} c^{p-4-r} x c^{r+1} d^{p-5-r} \in Y \text { for } 0 \leq r \leq p-5 .
$$

We prove (18) by induction on $r$. The case $r=0$ follows immediately from the identity $S_{p-1}\left(x a^{p-3}, x, x, c, c, \ldots, c\right) d^{p-5}=0$, using (4), (7), and (16). The remaining cases follow from the case $v=0$ and (9) in the same way as (8) follows from (6).

$$
x a^{p-3} x b^{p-4} x c^{p-4}+x a^{p-3} x c^{p-4} x b^{p-4} \in Y .
$$

If we set $u=b+c$ then (18) gives

$$
x a^{p-3} x b^{r} u^{p-4-r} x u^{r+1} b^{p-5-r} \in Y \text { for } 0 \leq r \leq p-5 .
$$

If we expand this and pick out the terms which are of degree $p-4$ in both $b$ and $c$, then we obtain

$(p-4-r) x a^{p-3} x b^{r+1} c^{p-5-r} x c^{r+1} b^{p-5-r}+(r+1) x a^{p-3} x b^{r} c^{p-4-r} x c^{r} b^{p-4-r} \in Y$ for $0 \leq r \leq p-5$. This gives (19).

$$
x b^{p-4} x c^{p-4} x a^{p-3} \in Y .
$$

Working modulo $Y$, and using (3), (4), (7), (16), and (19), we obtain

$$
\begin{aligned}
& x b^{p-4} x c^{p-4} x a^{p-3}=\left(x b^{p-4}\right)\left(x c^{p-4}\right) x a^{p-3} \\
&=\left(x b^{p-4}\right)\left(x c^{p-4}\right)\left(x a^{p-3}\right)+(p-3)\left(x b^{p-4}\right)\left(x c^{p-4}\right) a x a^{p-4} \\
&=-\left(x a^{p-3}\right)\left(\left(x b^{p-4}\right)\left(x c^{p-4}\right)\right)+(p-3)\left(x b^{p-4} a\right)\left(x c^{p-4}\right) x a^{p-4} \\
&+(p-3)\left(x b^{p-4}\right)\left(x c^{p-4} a\right) x a^{p-4} \\
&=-2 x a^{p-3} x b^{p-4} x c^{p-4}+(p-3) x b^{p-4} a x c^{p-4} x a^{p-4} \\
&-(p-3) x c^{p-4} a x b^{p-4} x a^{p-4} \\
&=-2 x a^{p-3} x b^{p-4} x c^{p-4}-(p-3) x b^{p-4} a x a^{p-4} x c^{p-4} \\
&+(p-3) x c^{p-4} a x a^{p-4} x b^{p-4} .
\end{aligned}
$$

Now we can use (8) and (19) to show that

$$
x b^{p-4} a x a^{p-4} x c^{p-4}=-\frac{1}{p-3} x a^{p-3} x b^{p-4} x c^{p-4}
$$

modulo $Y$, and to show that $x c^{p-4} a x a^{p-4} x b^{p-4}=-\frac{1}{p-3} x a^{p-3} x c^{p-4} x b^{p-4}=$ $\frac{1}{p-3} x a^{p-3} x b^{p-4} x c^{p-4}$ modulo $Y$; so $x b^{p-4} x c^{p-4} x a^{p-3} \in Y$, as required.

$$
x a^{p-3} x b^{p-4} x c^{p-4} \in Y \text {. }
$$

Using (7), (12), (16), and (20), the identity

$$
S_{p-1}\left(x a^{p-4}, x, x, a, b, b, \ldots, b\right) c^{p-4}=0
$$


gives

$$
\begin{gathered}
(p-3) x a^{p-4} x a b^{p-4} x c^{p-4}+x a^{p-3} x b^{p-4} x c^{p-4} \\
+(p-4) x a^{p-4} b x a b^{p-5} x c^{p-4} \in Y .
\end{gathered}
$$

Now, as we showed above, $x a^{p-4} x a b^{p-4} x c^{p-4}=\frac{2}{p-3} x a^{p-3} x b^{p-4} x c^{p-4}$. Also (6) gives $x a^{p-4} b x a b^{p-5} x c^{p-4}=-\frac{1}{p-3} x a^{p-3} x b^{p-4} x c^{p-4}$. So (21) follows from (22).

We are finally in a position to prove that the subspace $M$ defined above lies in $Y$.

$$
x a_{1} a_{2} \cdots a_{r} x b_{1} b_{2} \cdots b_{s} x c_{1} c_{2} \cdots c_{t} \in Y \quad \text { if } r+s+t=3 p-11 .
$$

Clearly an element of this form lies in the ideal generated by elements of the form $x d_{1} d_{2} \cdots d_{p-3}$. So we only need to consider the case when $r=p-3$. But then (7) and (16) imply that we may assume that $t \leq p-4$, and (4) implies that we may assume that $s \leq p-4$. This leaves only the case $s=t=p-4$, which is covered by $(21)$.

\section{ENGel- $n$ Lie Algebras $(n<p-1)$}

Let $L$ be a Lie algebra over a field $K$ of characteristic $p>5$, and let $L$ satisfy the Engel- $n$ identity for some $n<p-1$. We suppose that $L$ has an abelian subalgebra $A$, and an element $x$ such that $L$ is generated by $A$ and $x$. Just as in $\S 3$, the Engel- $n$ identity implies that $u v^{n}=0$ for all $u, v \in L$, and this is equivalent to the identity

$$
S_{n}\left(u, v_{1}, v_{2}, \ldots, v_{n}\right)=0,
$$

where

$$
S_{n}\left(u, v_{1}, v_{2}, \ldots, v_{n}\right)=\sum_{\sigma \in \operatorname{Sym}(n)} u v_{\sigma(1)} v_{\sigma(2)} \cdots v_{\sigma(n)}
$$

The proofs that follow are similar to the proofs in $\S 3$, but shorter and easier for the following reason. The identity $S_{n}(u, v, w, w, \ldots, w)=0$ implies

$$
\sum_{i=0}^{n-1} u w^{i} v w^{n-1-i}=0
$$

This gives

$$
\sum_{i=0}^{n-1} v\left(u w^{i}\right) w^{n-1-i}=0
$$

Expanding $u w^{i}$ in this identity gives

$$
\sum_{i=0}^{n-1} \sum_{r=0}^{i}(-1)^{r}\left(\begin{array}{l}
i \\
r
\end{array}\right) v w^{r} u w^{n-1-r}=0 .
$$

Interchanging the order of the summation we obtain

$$
\sum_{r=0}^{n-1} \sum_{i=r}^{n-1}(-1)^{r}\left(\begin{array}{l}
i \\
r
\end{array}\right) v w^{r} u w^{n-1-r}=0,
$$


and since $\sum_{i=r}^{n-1}\left(\begin{array}{l}i \\ r\end{array}\right)=\left(\begin{array}{c}n \\ r+1\end{array}\right)$ this finally gives us

$$
\sum_{r=0}^{n-1}(-1)^{r}\left(\begin{array}{c}
n \\
r+1
\end{array}\right) v w^{r} u w^{n-1-r}=0 .
$$

Note that we are assuming that $n<p-1$, so that the coefficients in this identity are different from those in (24). In the case when $n=p-1,\left(\begin{array}{c}n \\ r+1\end{array}\right)=(-1)^{r+1}$ for $0 \leq r \leq n-1$, and so identity (25) can be obtained from (24) by interchanging $u$ and $v$. We will use the identity $S_{n}\left(u, v_{1}, v_{2}, \ldots, v_{n}\right)=0$, together with (24) and (25) to establish a number of further identities that hold in $L$. In the identities that follow we let $a, b, c, a_{1}, a_{2}, \ldots, b_{1}, b_{2}, \ldots, c_{1}, c_{2}, \ldots$ denote arbitrary elements of the abelian Lie subalgebra $A$ of $L$. As in $\S 3$, we state each identity in turn, and follow each statement with a proof.

$$
u a_{1} a_{2} \cdots a_{n}=0 \text { for all } u \in L \text {. }
$$

This follows immediately from the identity $S_{n}\left(u, a_{1}, a_{2}, \ldots, a_{n}\right)=0$, using the fact that $a_{i} a_{j}=0$ for $1 \leq i, j \leq n$.

$$
x a^{n-2} x b^{n-1}=x a^{n-1} x b^{n-2}=0 .
$$

Identity (24) gives

and identity (25) gives

$$
\sum_{r=0}^{n-1} x a^{n-2} b^{r} x b^{n-1-r}=0,
$$

$$
\sum_{r=0}^{n-1}(-1)^{r}\left(\begin{array}{c}
n \\
r+1
\end{array}\right) x a^{n-2} b^{r} x b^{n-1-r}=0 .
$$

Now (26) implies that $x a^{n-2} b^{r}=0$ if $r \geq 2$, and so these two identities imply that

$$
x a^{n-2} x b^{n-1}=x a^{n-2} b x b^{n-2}=0 .
$$

Similarly, (24) and (25) give

$$
\begin{gathered}
\sum_{r=0}^{n-1} x a^{r} x a^{n-1-r} b^{n-2}=0, \\
\sum_{r=0}^{n-1}(-1)^{r}\left(\begin{array}{c}
n \\
r+1
\end{array}\right) x a^{r} x a^{n-1-r} b^{n-2}=0,
\end{gathered}
$$

and these identities, together with (26), imply that

$$
x a^{n-1} x b^{n-2}=x a^{n-2} x a b^{n-2}=0 .
$$

This establishes (27).

Now let $Y$ be the ideal of $L$ generated by elements of the form $x a_{1} a_{2} \cdots a_{n-1}$.

$$
x a^{n-4} b^{r} x b^{n-1-r} \in Y \text { for } 0 \leq r \leq n-1 .
$$

Clearly $x a^{n-4} b^{r} x b^{n-1-r} \in Y$ for $r \geq 3$. So (24) and (25) give

$$
\begin{gathered}
x a^{n-4} x b^{n-1}+x a^{n-4} b x b^{n-2}+x a^{n-4} b^{2} x b^{n-3} \in Y, \\
n x a^{n-4} x b^{n-1}-\left(\begin{array}{c}
n \\
2
\end{array}\right) x a^{n-4} b x b^{n-2}+\left(\begin{array}{l}
n \\
3
\end{array}\right) x a^{n-4} b^{2} x b^{n-3} \in Y .
\end{gathered}
$$


Also the fact that $x a^{n-4}\left(x b^{n-1}\right) \in Y$ gives

$$
x a^{n-4} x b^{n-1}-(n-1) x a^{n-4} b x b^{n-2}+\left(\begin{array}{c}
n-1 \\
2
\end{array}\right) x a^{n-4} b^{2} x b^{n-3} \in Y .
$$

These three identities establish (28) since

$$
\left|\begin{array}{ccc}
1 & 1 & 1 \\
n & -\left(\begin{array}{c}
n \\
2
\end{array}\right) & \left(\begin{array}{c}
n \\
3
\end{array}\right) \\
1 & -(n-1) & \left(\begin{array}{c}
n-1 \\
2
\end{array}\right)
\end{array}\right|=-\frac{n^{2}\left(n^{2}-1\right)}{12} \neq 0 .
$$

(29) $x a_{1} a_{2} \cdots a_{r} b^{s} x b^{n-1-s} c_{1} c_{2} \cdots c_{t} \in Y$ for $0 \leq s \leq n-1$ if $r+t=n-4$.

This follows from (28) in the same way as we showed in $\S 3$ that (8) follows from (6).

$$
x a^{n-2} x b^{n-3} \in Y .
$$

Using (28) we see that the identity $S_{n}(b, x, x, a, a, \ldots, a) b^{n-4}=0$ implies that

$$
b x a^{n-4} x a^{2} b^{n-4}+b x a^{n-3} x a b^{n-4} \in Y .
$$

Now $b x a^{n-4} x a^{2} b^{n-4}=-x b a^{n-4} x a^{2} b^{n-4}$. If we set $u=a+b$, then (29) implies that $x u^{n-3} x u^{2} b^{n-4} \in Y$. Expanding, and picking out the terms which are of degree $n-2$ in $a$ and of degree $n-3$ in $b$, we obtain

$$
(n-3) x b a^{n-4} x a^{2} b^{n-4}+2 x a^{n-3} x a b^{n-3} \in Y .
$$

Similarly $b x a^{n-3} x a b^{n-4}=-x b a^{n-3} x a b^{n-4}=\frac{1}{n-2} x a^{n-2} x b^{n-3}$. So (31) gives

$$
\frac{2}{n-3} x a^{n-3} x a b^{n-3}+\frac{1}{n-2} x a^{n-2} x b^{n-3} \in Y \text {. }
$$

Now if $n$ is odd, then $n-3$ is even. So if we let $s=(n-3) / 2$ then

$$
0=\left(x a^{s}\right)\left(x a^{s}\right)=\sum_{r=0}^{s}(-1)^{r}\left(\begin{array}{l}
s \\
r
\end{array}\right) x a^{s+r} x a^{s-r} .
$$

So

$$
x a^{n-3} x a b^{n-3}=-\sum_{r=0}^{s-1}(-1)^{r}\left(\begin{array}{l}
s \\
r
\end{array}\right) x a^{s+r} x a^{s-r+1} b^{n-3} \in Y .
$$

Hence (32) implies that $x a^{n-2} x b^{n-3} \in Y$. On the other hand if $n$ is even, then $n-2$ is even, and so if we let $s=(n-2) / 2$ then $\left(x a^{s}\right)\left(x a^{s}\right)=0$, and expanding this we obtain

$$
x a^{n-2} x b^{n-3}-\frac{n-2}{2} x a^{n-3} x a b^{n-3} \in Y .
$$

Together with (32) this implies that $x a^{n-2} x b^{n-3} \in Y$. So, whether $n$ is even or odd, we have shown that $x a^{n-2} x b^{n-3} \in Y$.

\section{THE IDEAL GeNERATED BY AN ELEMENT}

Let $L$ be an Engel- $n$ Lie algebra over a field $K$ with $\operatorname{char}(K)>n$, and let $x$ be an element of $L$. In this section we study the ideal $I d_{L}(x)$ of $L$ generated by $x$. Clearly, $I d_{L}(x)$ is spanned by products in $L$ such that at 
least one of the elements in the product is $x$. More generally, it is easy to see that $\left(I d_{L}(x)\right)^{m}$ is spanned by products in $L$ such that $x$ occurs at least $m$ times in the product. Let $u$ be a product of $k$ elements $v_{1}, v_{2}, \ldots, v_{k} \in L$, in some order with some bracketing. If $x$ occurs $m$ times in the sequence $v_{1}, v_{2}, \ldots, v_{k}$, then we say that $u$ has type $(m ; k)$. Note that the elements $v_{1}, v_{2}, \ldots, v_{k}$ could also be products, and that some of these products might involve $x$, so that type $(m ; k)$ depends on the particular expression for $u$ as a product of $v_{1}, v_{2}, \ldots, v_{k}$. So, strictly speaking, we should call $(m ; k)$ the type of the expression for $u$, rather than the type of $u$. Our aim in this section is to establish the following three lemmas.

Lemma 4. If $m>2$, then $\left(I d_{L}(x)\right)^{m}$ is generated as an ideal by products of type $(m ; k)$ where $k \leq m+1+\frac{(m-1)(2 n-5)}{2}$.

Lemma 5. Let $m>2$, and let

$$
v=x a_{11} a_{12} \cdots a_{1 k_{1}} x a_{21} a_{22} \cdots a_{2 k_{2}} x \cdots x a_{m 1} a_{m 2} \cdots a_{m k_{m}}
$$

be a product of type $(m ; k)$ in $L$. Let $Y$ be the ideal of $L$ generated by elements of the form $x a_{1} a_{2} \cdots a_{n-1}$ where $a_{i} \in\left\{a_{r s} \mid 1 \leq r \leq m, 1 \leq s \leq k_{r}\right\}$ for $i=1,2, \ldots, n-1$, and let $Z$ be the ideal of $L$ generated by all products in $L$ of type $(m ; r)$ with $r<k$. If $k \geq m+2+\frac{(m-1)(3 m-10)}{3}$ then $v \in Y+Z$.

Lemma 6. If $m>2$, then $\left(I d_{L}(x)\right)^{m}$ is contained in the ideal generated by products of type $(r ; k)$ where $\frac{3 m+3}{8} \leq r \leq m$ and $k<r+2+\frac{(r-1)(3 n-10)}{3}$.

Let $L$ be an Engel- $n$ Lie algebra over a field $K$ with $\operatorname{char}(K)>n$, and let $x$ be an element of $L$. Then $I d_{L}(x)$ is spanned by elements of the form $x a_{1} a_{2} \ldots a_{k}$ with $a_{1}, a_{2}, \ldots, a_{k} \in L$, and with $k \geq 0$. So $\left(\operatorname{Id}_{L}(x)\right)^{m}$ is spanned by elements of the form

$$
\left(x a_{11} a_{12} \cdots a_{1 k_{1}}\right)\left(x a_{21} a_{22} \cdots a_{2 k_{2}}\right) \cdots\left(x a_{m 1} a_{m 2} \cdots a_{m k_{m}}\right) .
$$

Note that this element has type $(m ; k)$ where $k=m+k_{1}+k_{2}+\cdots+k_{m}$. We want to show that $\left(I d_{L}(x)\right)^{m}$ is generated as an ideal by elements of type $(m ; k)$ where $k \leq m+1+\frac{(m-1)(2 n-5)}{2}$. So we let $Z$ be the ideal of $L$ generated by these elements, and we prove by induction on $k$ that elements of type $(m ; k)$ lie in $Z$ for all $k$. We let $u$ be a product of type $(m ; k)$ for some $k>$ $m+1+\frac{(m-1)(2 n-5)}{2}$ and we suppose that all products of type $(m ; r)$ lie in $Z$ for all $r<k$. The product $u$ can be written as a linear combination of products of type $(m ; k)$ of the form

$$
x a_{11} a_{12} \cdots a_{1 k_{1}} x a_{21} a_{22} \cdots a_{2 k_{2}} x \cdots x a_{m 1} a_{m 2} \cdots a_{m k_{m}}
$$

(where $m+k_{1}+k_{2}+\cdots+k_{m}=k$ ), and so it is sufficient to prove that

$$
x a_{11} a_{12} \cdots a_{1 k_{1}} x a_{21} a_{22} \cdots a_{2 k_{2}} x \cdots x a_{m 1} a_{m 2} \cdots a_{m k_{m}} \in Z
$$

for all $a_{i j} \in L$. Clearly it is sufficient to prove this in the case when $L$ is the relatively free Engel- $n$ Lie algebra freely generated by $x, a_{11}, a_{12}, \ldots, a_{m k_{m}}$. Now we let $J$ be the ideal of $L$ generated by $\left\{a_{i j} a_{r s} \mid 1 \leq i, r \leq m, 1 \leq j \leq\right.$ $\left.k_{i}, 1 \leq s \leq k_{r}\right\}$. If we can show that

$$
v=x a_{11} a_{12} \cdots a_{1 k_{1}} x a_{21} a_{22} \cdots a_{2 k_{2}} x \cdots x a_{m 1} a_{m 2} \cdots a_{m k_{m}} \in Z+J
$$


then, by an argument similar to the argument used in the proof of Lemma 1, it follows that $v \in Z$. Since $L, Z$, and $J$ are all multigraded, if $v \in Z+J$ then $v$ can be expressed in the form $v=b+c$, where $b \in Z$ and $c \in J$, and where $b$ and $c$ have the same multiweight as that of $v$ in the free generators of $L$. But then $c$ can be written as a linear combination of products of the form $\left(a_{i j} a_{r s}\right) v_{3} v_{4} \cdots v_{k}$ where $v_{3}, v_{4}, \ldots, v_{k}$ are free generators of $L$, and where $x$ occurs $m$ times in the sequence $v_{3}, v_{4}, \ldots, v_{k}$. If we treat $a_{i j} a_{r s}$ as a single element of $L$ then $\left(a_{i j} a_{r s}\right) v_{3} v_{4} \cdots v_{k}$ has type $(m ; k-1)$ and so lies in $Z$ by the inductive hypothesis. Hence $v \in Z$ as required.

So from now on we work modulo the ideal $Z+J$. In other words, we assume that all products of type $(m ; r)$ are zero for all $r<k$, and we assume that $a_{i j} a_{r s}=0$ for all $i, j, r, s$ such that $1 \leq i, r \leq m, 1 \leq j \leq k_{i}, 1 \leq s \leq k_{r}$. We make one further inductive hypothesis. If

$$
w=x b_{11} b_{12} \cdots b_{1 r_{1}} x b_{21} b_{22} \cdots b_{2 r_{2}} x \cdots x b_{m 1} b_{m 2} \cdots b_{m r_{m}}
$$

is a product of type $(m ; k)$ with $b_{i j} \in L$ for all $i, j$ such that $1 \leq i \leq m$, $1 \leq j \leq r_{i}$, and with $m+r_{1}+r_{2}+\cdots+r_{m}=k$, then we define the index of $w$ to be $\left(r_{1}, r_{2}, \ldots, r_{m}\right)$. We order the set of possible indexes lexicographically, so that

$$
\left(r_{1}, r_{2}, \ldots, r_{m}\right)>\left(k_{1}, k_{2}, \ldots, k_{m}\right)
$$

if for some $s$ with $1 \leq s<m$ we have $r_{i}=k_{i}$ for $1 \leq i<s$ and $r_{s}>k_{s}$. We make the additional inductive hypothesis that $w=0$ for all such $w$ with index $\left(r_{1}, r_{2}, \ldots, r_{m}\right)>\left(k_{1}, k_{2}, \ldots, k_{m}\right)$.

Now we show that these assumptions imply that $v=0$. First note that if $k_{m}>0$ then

$$
u=x a_{11} a_{12} \cdots a_{1 k_{1}} x a_{21} a_{22} \cdots a_{2 k_{2}} x \cdots x a_{m 1} a_{m 2} \cdots a_{m k_{m}-1}
$$

has type $(m ; k-1)$. The inductive hypothesis implies that $u=0$, and so $v=u a_{m k_{m}}=0$. Next, note that we can use the results of $\S \S 3$ and 4 since $a_{i j} a_{r s}=0$ for all $i, j, r, s$ such that $1 \leq i, r \leq m, 1 \leq j \leq k_{i}, 1 \leq s \leq k_{r}$. Identities (1) and (26) imply that $v=0$ if $k_{r} \geq n$ for any $r$ with $1 \leq r<m$. We can also show that $v=0$ if $k_{r}=n-1$ for some $r$ with $1<r<m$. To see this suppose that $1<r<m$ and that $k_{r}=n-1$. Set

$$
u=x a_{11} a_{12} \cdots a_{1 k_{1}} x a_{21} a_{22} \cdots a_{2 k_{2}} x \cdots x a_{r-11} a_{r-12} \cdots a_{r-1 k_{r-1}},
$$

and use the identity

$$
S_{n}\left(u, x, a_{r 1}, a_{r 2}, \ldots, a_{r n-1}\right) x a_{r+11} a_{r+12} \cdots a_{r+1 k_{r+1}} x \cdots x a_{m 1} a_{m 2} \cdots a_{m k_{m}}=0 \text {. }
$$

This identity enables us to express $v$ as a sum of products of type $(m ; k)$ with higher index than $v$. So $v=0$. Identities (2) and (27) imply that $v=0$ if $k_{1}=n-1$ and $k_{2}=n-2$. Identities (2) and (27) can also be used to show that $v=0$ if $k_{r}=k_{r+1}=n-2$ for some $r$ with $1<r<m$. For they imply that if $x, u, a_{1}, a_{2}, \ldots, a_{n-2}, b_{1}, b_{2}, \ldots, b_{n-2}$ are elements of an Engel- $n$ Lie algebra over $K$ then $u x a_{1} a_{2} \cdots a_{n-2} x b_{1} b_{2} \cdots b_{n-2}=-x u a_{1} a_{2} \cdots a_{n-2} x b_{1} b_{2} \cdots b_{n-2}$ lies in the ideal generated by the elements $u a_{i}, u b_{i}, a_{i} b_{j}(1 \leq i, j \leq n-2)$. If $k_{r}=k_{r+1}=n-2$ for some $r$ with $1<r<m$ then we substitute

$$
x a_{11} a_{12} \cdots a_{1 k_{1}} x a_{21} a_{22} \cdots a_{2 k_{2}} x \cdots x a_{r-11} a_{r-12} \cdots a_{r-1 k_{r-1}}
$$


for $u$, and substitute $a_{r j}$ for $a_{j}$ and $a_{r+1 j}$ for $b_{j}(1 \leq j \leq n-2)$ in this identity. The identity then implies that $v$ lies in the ideal of $L$ generated by elements of the form

$$
x a_{11} a_{12} \cdots a_{1 k_{1}} x a_{21} a_{22} \cdots a_{2 k_{2}} x \cdots x a_{r-11} a_{r-12} \cdots a_{r-1 k_{r-1}} a_{i j}
$$

$(i=r, r+1,1 \leq j \leq n-2)$. Since $L$ is multigraded this implies that $v$ can be expressed as a linear combination of products of type $(m ; k)$ all of which have higher index than $v$. By the inductive hypothesis, all products with higher index than $v$ are zero, and so $v=0$. Finally note that if $k_{m-1}=n-2$ then setting

$$
u=x a_{11} a_{12} \cdots a_{1 k_{1}} x a_{21} a_{22} \cdots a_{2 k_{2}} x \cdots x a_{m-21} a_{m-22} \cdots a_{m-2 k_{m-2}}
$$

we see that the identity $S_{n}\left(u, x, x, a_{m-11}, a_{m-12}, \ldots, a_{m-1 n-2}\right)=0$ enables us to express $v$ as a linear combination of products some of which have type $(m ; k)$ but have higher index than that of $v$, and some of which lie in the ideal generated by products of type $(m ; k-1)$. So $v=0$.

To summarize, $v=0$ unless the following conditions are satisfied:

$$
\begin{gathered}
k_{1}<n, \\
k_{r}<n-1 \text { for } 1<r<m-1, \\
k_{m-1}<n-2, \\
k_{m}=0, \\
k_{1}<n-1 \text { or } k_{2}<n-2, \\
\text { If } 1<r<m \text { then } k_{r}<n-2 \text { or } k_{r+1}<n-2 .
\end{gathered}
$$

But if all these conditions are satisfied then $k=m+k_{1}+k_{2}+\cdots+k_{m} \leq$ $m+1+\frac{(m-1)(2 n-5)}{2}$, contrary to the initial assumption. So $v=0$ as required. This completes the proof of Lemma 4.

Now we prove Lemma 5 . Let $m>2$, and let

$$
v=x a_{11} a_{12} \cdots a_{1 k_{1}} x a_{21} a_{22} \cdots a_{2 k_{2}} x \cdots x a_{m 1} a_{m 2} \cdots a_{m k_{m}}
$$

be a product of type $(m ; k)$ in $L$. Let $Y$ be the ideal of $L$ generated by elements of the form $x a_{1} a_{2} \cdots a_{n-1}$ where $a_{i} \in\left\{a_{r s} \mid 1 \leq r \leq m, 1 \leq s \leq k_{r}\right\}$ for $i=1,2, \ldots, n-1$, and let $Z$ be the ideal of $L$ generated by all products in $L$ of type $(m ; r)$ with $r<k$. We show that if $k \geq m+2+\frac{(m-1)(3 m-10)}{3}$ then $v \in Y+Z$. As above, we let $J$ be the ideal of $L$ generated by $\left\{a_{i j} a_{r s} \mid 1 \leq\right.$ $\left.i, r \leq m, 1 \leq j \leq k_{i}, 1 \leq s \leq k_{r}\right\}$. Just as in the proof of Lemma 4 we see that we can assume that $L$ is multigraded, and that $Y=Z=J=\{0\}$. We suppose that $k \geq m+2+\frac{(m-1)(3 m-10)}{3}$ and we show that this (together with the assumption $Y=Z=J=\{0\}$ ) implies that $v=0$. We can assume by induction that $w=0$ if

$$
w=x b_{11} b_{12} \cdots b_{1 r_{1}} x b_{21} b_{22} \cdots b_{2 r_{2}} x \cdots x b_{m 1} b_{m 2} \cdots b_{m r_{m}}
$$

is a product of type $(m ; k)$ with $b_{i j} \in\left\{a_{r s} \mid 1 \leq r \leq m, 1 \leq s \leq k_{r}\right\}$ for $i=$ $1,2, \ldots, m, j=1,2, \ldots, r_{i}$, and with $\left(r_{1}, r_{2}, \ldots, r_{m}\right)>\left(k_{1}, k_{2}, \ldots, k_{m}\right)$.

As in the proof of Lemma 4, we see that $v=0$ if $k_{r} \geq n-1$ for some $r$ with $1<r<m$, or if $k_{m}>0$. Since we are making the assumption that $Y=\{0\}$, it is clear that $v=0$ if $k_{1} \geq n-1$. The assumption that $Y=\{0\}$ also means 
that we can make use of identities (3), (4), and (23) from $\S 3$ and identity (30) from $\S 4$. We show that these identities imply that $v=0$ if $k_{r}=n-2$ for any $r$ with $1<r<m$.

First suppose that $k_{2}=n-2$. If $k_{1}=n-2$ then $v=0$ by (4) and (30). If $k_{1}<n-2$ then, using the fact that products of type $(m ; k)$ are zero if they have index greater than $\left(k_{1}, k_{2}, \ldots, k_{m}\right)$, we see that

$$
\begin{aligned}
v & =x a_{11} a_{12} \cdots a_{1 k_{1}} x a_{21} a_{22} \cdots a_{2 k_{2}} x \cdots x a_{m 1} a_{m 2} \cdots a_{m k_{m}} \\
& =\left(x a_{11} a_{12} \cdots a_{1 k_{1}}\right)\left(x a_{21} a_{22} \cdots a_{2 k_{2}}\right) x \cdots x a_{m 1} a_{m 2} \cdots a_{m k_{m}} \\
& =-\left(x a_{21} a_{22} \cdots a_{2 k_{2}}\right)\left(x a_{11} a_{12} \cdots a_{1 k_{1}}\right) x \cdots x a_{m 1} a_{m 2} \cdots a_{m k_{m}} \\
& =0 .
\end{aligned}
$$

Next suppose that $k_{r}=n-2$ for some $r$ with $2<r<m$, and let $u=$ $x a_{11} a_{12} \cdots a_{1 k_{1}} x a_{21} a_{22} \cdots a_{2 k_{2}} x \cdots x a_{r-21} a_{r-22} \cdots a_{r-2 k_{r-2}}$. If $k_{r-1}=0$ then the identity

$$
\begin{aligned}
& S_{n}\left(u, x, x, a_{r 1}, a_{r 2}, \ldots, a_{r n-2}\right) \\
& \quad \cdot x a_{r+11} a_{r+12} \cdots a_{r+1 k_{r+1}} x \cdots x a_{m 1} a_{m 2} \cdots a_{m k_{m}}=0
\end{aligned}
$$

enables us to express $v$ as a linear combination of products of type $(m ; k)$ with index greater than $\left(k_{1}, k_{2}, \ldots, k_{m}\right)$. Hence $v=0$ if $k_{r-1}=0$. Now suppose that $k_{r-1}=s>0$, and let $b, c$ be elements in the linear span of $\left\{a_{i j} \mid 1 \leq i \leq m, 1 \leq j \leq k_{i}\right\}$. Using the fact that products of type $(m ; k)$ are zero if they have index greater than $\left(k_{1}, k_{2}, \ldots, k_{m}\right)$, the identity

$$
S_{n}\left(u x b^{s-1}, x, c, c, \ldots, c\right) x a_{r+11} a_{r+12} \cdots a_{r+1 k_{r+1}} x \cdots x a_{m 1} a_{m 2} \cdots a_{m k_{m}}=0
$$

gives

$$
\begin{aligned}
& u x b^{s-1} x c^{n-1} x a_{r+11} \cdots x a_{m 1} a_{m 2} \cdots a_{m k_{m}} \\
& +u x b^{s-1} c x c^{n-2} x a_{r+11} \cdots x a_{m 1} a_{m 2} \cdots a_{m k_{m}}=0 .
\end{aligned}
$$

Similarly the identity

$$
u x b^{s-1}\left(x c^{n-1}\right) x a_{r+11} a_{r+12} \cdots a_{r+1 k_{r+1}} x \cdots x a_{m 1} a_{m 2} \cdots a_{m k_{m}}=0
$$

gives

$$
\begin{aligned}
& u x b^{s-1} x c^{n-1} x a_{r+11} \cdots x a_{m 1} a_{m 2} \cdots a_{m k_{m}} \\
& -(n-1) u x b^{s-1} c x c^{n-2} x a_{r+11} \cdots x a_{m 1} a_{m 2} \cdots a_{m k_{m}}=0 .
\end{aligned}
$$

So

$$
\begin{aligned}
& u x b^{s-1} x c^{n-1} x a_{r+11} \cdots x a_{m 1} a_{m 2} \cdots a_{m k_{m}} \\
& \quad=u x b^{s-1} c x c^{n-2} x a_{r+11} \cdots x a_{m 1} a_{m 2} \cdots a_{m k_{m}}=0 .
\end{aligned}
$$

Linearizing this we see that

$$
u x b_{1} b_{2} \cdots b_{s-1} x c_{1} c_{2} \cdots c_{n-1} x a_{r+11} \cdots x a_{m 1} a_{m 2} \cdots a_{m k_{m}}=0
$$

and

$$
u x b_{1} b_{2} \cdots b_{s-1} c x c^{n-2} x a_{r+11} \cdots x a_{m 1} a_{m 2} \cdots a_{m k_{m}}=0
$$

for all $b_{1}, b_{2}, \ldots, b_{s-1}, c_{1}, c_{2}, \ldots, c_{n-1}, c$ in the linear span of $\left\{a_{i j} \mid 1 \leq i \leq\right.$ $\left.m, 1 \leq j \leq k_{i}\right\}$. If we let $0 \leq t \leq s-1$, then (34) gives

$$
u x b^{t} c^{s-1-t}(b+c) x(b+c)^{n-2} x a_{r+11} \cdots x a_{m 1} a_{m 2} \cdots a_{m k_{m}}=0 \text {. }
$$


Expanding, and picking out the terms of degree $s$ in $b$ and of degree $n-2$ in $c$, we obtain

$$
\begin{aligned}
& \left(\begin{array}{c}
n-2 \\
s-t
\end{array}\right) u x b^{t} c^{s-t} x b^{s-t} c^{n-2-s+t} x a_{r+11} \cdots x a_{m 1} a_{m 2} \cdots a_{m k_{m}} \\
& \quad+\left(\begin{array}{c}
n-2 \\
s-t-1
\end{array}\right) u x b^{t+1} c^{s-1-t} x b^{s-1-t} c^{n-1-s+t} x a_{r+11} \cdots x a_{m 1} a_{m 2} \cdots a_{m k_{m}}=0 .
\end{aligned}
$$

These identities for $t=0,1, \ldots, s-1$ show that

$$
u x b^{s} x c^{n-2} x a_{r+11} \cdots x a_{m 1} a_{m 2} \cdots a_{m k_{m}}
$$

is a nonzero multiple of

$$
u x c^{s} x c^{n-2-s} b^{s} x a_{r+11} \cdots x a_{m 1} a_{m 2} \cdots a_{m k_{m}} .
$$

Now consider the identity

$$
S_{n}(u, x, x, c, c, \ldots, c) b^{s} x a_{r+11} a_{r+12} \cdots a_{r+1 k_{r+1}} x \cdots a_{m 1} a_{m 2} \cdots a_{m k_{m}}=0 \text {. }
$$

Expanding this, using (33), and ignoring terms which have index greater than $\left(k_{1}, k_{2}, \ldots, k_{m}\right)$, we obtain

$$
u x c^{s} x c^{n-2-s} b^{s} x a_{r+11} a_{r+12} \cdots a_{r+1 k_{r+1}} x \cdots x a_{m 1} a_{m 2} \cdots a_{m k_{m}}=0 .
$$

By the above remarks, this implies that

$$
u x b^{s} x c^{n-2} x a_{r+11} a_{r+12} \cdots a_{r+1 k_{r+1}} x \cdots x a_{m 1} a_{m 2} \cdots a_{m k_{m}}=0,
$$

and linearizing we obtain $v=0$ as required.

So far, we have shown that $v=0$ unless

$$
\begin{gathered}
k_{1} \leq n-2, \\
k_{r} \leq n-3 \text { for } r=2,3, \ldots, m-1, \\
k_{m}=0 .
\end{gathered}
$$

If these conditions are satisfied then $k_{1}+k_{2}+k_{3} \leq 3 n-8$, and $k_{r}+k_{r+1}+k_{r+2} \leq$ $3 n-9$ for $r=2,3, \ldots, m-3$. Identities (23) and (30) imply that $v=0$ if $k_{1}+k_{2}+k_{3}=3 n-8$. They also imply that $v=0$ if $k_{r}+k_{r+1}+k_{r+2}=3 n-9$ for some $r$ with $2 \leq r \leq m-3$. To see this, let $2 \leq r \leq m-3$ and let $k_{r}+k_{r+1}+k_{r+2}=3 n-9$. Then let

$$
u=x a_{11} a_{12} \cdots a_{1 k_{1}} x a_{21} a_{22} \cdots a_{2 k_{2}} x \cdots x a_{r-11} a_{r-12} \cdots a_{r-1 k_{r-1}} .
$$

Identities (23) and (30), together with the above calculations, show that

$$
\begin{aligned}
v & =u x a_{r 1} a_{r 2} \cdots a_{r k_{r}} x \cdots x a_{m 1} a_{m 2} \cdots a_{m k_{m}} \\
& =-x u a_{r 1} a_{r 2} \cdots a_{r k_{r}} x \cdots x a_{m 1} a_{m 2} \cdots a_{m k_{m}}
\end{aligned}
$$

lies in the ideal generated by elements of the form $b c$ and $x b_{1} b_{2} \cdots b_{n-1}$, where $b, c, b_{1}, b_{2}, \ldots, b_{n-1}$ are elements of the set $\left\{u, a_{r 1}, a_{r 2}, \ldots, a_{m k_{m}}\right\}$. Now, by assumption, $b c=0$ if $b, c \in\left\{a_{r 1}, a_{r 2}, \ldots, a_{m k_{m}}\right\}$, and $x b_{1} b_{2} \cdots b_{n-1}=$ 0 if $b_{1}, b_{2}, \ldots, b_{n-1} \in\left\{a_{r 1}, a_{r 2}, \ldots, a_{m k_{m}}\right\}$. So, using the fact that $L$ is multigraded, we see that $v$ lies in the ideal generated by elements of the form $u c$ and $u x b_{1} b_{2} \cdots b_{n-2}$ where $c, b_{1}, b_{2}, \ldots, b_{n-2} \in\left\{a_{r 1}, a_{r 2}, \ldots, a_{m k_{m}}\right\}$. Since $L$ is multigraded, this implies that $v$ is a linear combination of products of type $(m ; k)$ of the form $u c v_{1} v_{2} \cdots v_{s}$ and of the form $u x b_{1} b_{2} \cdots b_{n-2} w_{1} w_{2} \cdots w_{t}$ 
where $v_{1}, v_{2}, \ldots, v_{s}, w_{1}, w_{2}, \ldots, w_{t} \in\left\{x, a_{r 1}, a_{r 2}, \ldots, a_{m k_{m}}\right\}$. Note that we have shown above that $v=0$ if $k_{r}=n-2$. So we may assume that $k_{r}<n-2$, which implies that all these products have index greater than that of $v$. So $v=0$ as required.

Putting all this together we have shown that $v=0$ unless

$$
\begin{gathered}
k_{1} \leq n-2, \\
k_{r} \leq n-3 \text { for } r=1,2, \ldots, m-1, \\
k_{m}=0 \\
k_{1}+k_{2}+k_{3} \leq 3 n-9, \\
k_{r}+k_{r+1}+k_{r+2} \leq 3 n-10 \text { for } r=2,3, \ldots, m-3 .
\end{gathered}
$$

But if all these conditions are satisfied then $k=m+k_{1}+k_{2}+\cdots+k_{m}<$ $m+2+\frac{(m-1)(3 n-10)}{3}$, contrary to our initial assumptions. So $v=0$ in every case. This completes the proof of Lemma 5.

Finally we prove Lemma 6 . We let $x$ be an element of an Engel- $n$ Lie algebra $L$ over $K$, we let $m>2$, and we let $T$ be the ideal of $L$ generated by products of type $(r ; k)$ where $\frac{3 m+3}{8} \leq r \leq m$ and $k<r+2+\frac{(r-1)(3 n-10)}{3}$. We need to show that $\left(I d_{L}(x)\right)^{m} \leq T$.

By Lemma 4, $\left(I d_{L}(x)\right)^{m}$ is generated as an ideal by products of type $(m ; k)$ with $k \leq m+1+\frac{(m-1)(2 n-5)}{2}$, and we show that all these products lie in $T$. So let

$$
v=x a_{11} a_{12} \cdots a_{1 k_{1}} x a_{21} a_{22} \cdots a_{2 k_{2}} x a_{31} \cdots x a_{m 1 m 2} \cdots a_{m k_{m}}
$$

be a product of type $(m ; k)$, where $k \leq m+1+\frac{(m-1)(2 n-5)}{2}$, and suppose by induction that all products of type $(m ; r)$ lie in $T$ for all $r<k$. If $k<$ $m+2+\frac{(m-1)(3 n-10)}{3}$ then $v \in T$, and we are done. If $k \geq m+2+\frac{(m-1)(3 n-10)}{3}$, then $v \in Y+Z$ by Lemma 5 , where $Z$ is the ideal generated by products of type $(m ; r)$ with $r<k$. By our inductive hypothesis, $Z \leq T$. So $v=b+c$ for some $b \in Y$ and some $c \in T$. As usual, we may assume that $b$ and $c$ have the same multiweight as $v$. So $b$ can be written as a linear combination of products of type $(m ; k)$ of the form

$$
x a_{1} a_{2} \cdots a_{n-1} v_{n+1} v_{n+2} \cdots v_{k}
$$

where $a_{1}, a_{2}, \ldots, a_{n-1} \in\left\{a_{i j} \mid 1 \leq i \leq m, 1 \leq j \leq k_{i}\right\}$ and $v_{n+1}, v_{n+2}, \ldots, v_{k}$ $\in\{x\} \cup\left\{a_{i j} \mid 1 \leq i \leq m, 1 \leq j \leq k_{i}\right\}$. Treating $x a_{1} a_{2} \cdots a_{n-1}$ as a single element of $L$, we see that $x a_{1} a_{2} \cdots a_{n-1} v_{n+1} v_{n+2} \cdots v_{k}=\left(x a_{1} a_{2} \cdots a_{n-1}\right) v_{n+1} v_{n+2} \cdots v_{k}$ can be viewed as a product of type $(m-1 ; k-n+1)$. So $v$ can be written modulo $T$ as a linear combination of products of type $(m-1 ; k-n+1)$. We now want to repeat the argument and show that products of type $(m-$ $1 ; k-n+1)$ can be written modulo $T$ as linear combinations of products of type $(m-2 ; k-2 n+2)$. But to do this we need to be able to assume that all products of type $(m-1 ; r)$ with $r<k-n+1$ lie in $T$. So we extend our inductive hypothesis to include the assumption that all products of type $(r ; s)$ lie in $T$ for all pairs $r, s$ with $\frac{3 m+3}{8} \leq r \leq m$ and $s<k-(m-r)(n-1)$. Then by repeated use of the argument above we see that if $\frac{3 m+3}{8} \leq r \leq m$ then $v$ can be written modulo $T$ as a linear combination of products of type $(r ; k-(m-r)(n-1))$. Now $k \leq m+1+\frac{(m-1)(2 n-5)}{2}$, and so $k-(m-r)(n-1) \leq$ 
$m+1+\frac{(m-1)(2 n-5)}{2}-(m-r)(n-1)=\frac{7}{2}-\frac{m}{2}-r+(r-1) n$. If we choose $r$ minimal subject to the condition $r \geq \frac{3 m+3}{8}$ then $r \leq \frac{3 m+10}{8}$, and so $m \geq \frac{8 r-10}{3}$. Hence $k-(m-r)(n-1) \leq \frac{7}{2}-\frac{8 r-10}{6}-r+(r-1) n<r+2+\frac{(r-1)(3 n-10)}{3}$. But this implies that products of type $(r ; k-(m-r)(n-1))$ all lie in $T$. So $v \in T$ as required, and this completes the proof of Lemma 6.

\section{ENGEL-6 LiE ALgEBRAS OF CHARACTERISTIC 7}

In this section we prove the following theorem.

Theorem 7. If $L$ is an m-generator Engel-6 Lie algebra of characteristic 7 then $L$ is nilpotent of class at most $51 \mathrm{~m}^{8}$.

As an immediate corollary we obtain a polynomial bound on the class of a finite $m$-generator group of exponent 7 .

Theorem 8. If $G$ is a finite m-generator group of exponent 7 then $G$ is nilpotent of class at nost $51 \mathrm{~m}^{8}$.

I made use of the nilpotent quotient algorithm for graded Lie rings described in Havas, Newman, and Vaughan-Lee [4] to compute the Lie algebra $M$ described in $\S 2$. The computations showed that if $n=6$ and $\operatorname{char}(K)=7$ then $M$ is nilpotent of class 22 . In addition, the computations showed that if $v$ is a product with multiweight $\left(w_{0}, w_{1}, w_{2}, \ldots\right)$ in the generators $\tilde{x}, \tilde{a}_{1}, \tilde{a}_{2}, \ldots$ of $M$, then $v=0$ if $w_{0}-\sum_{i>0} w_{i}>10$. So, using Theorem 3 and the remark that follows immediately after its proof, we see that if $a, b$ are elements of an Engel-6 Lie algebra of characteristic 7, then the ideal generated by $a b^{4}$ is nilpotent of class at most 73 . We can actually do slightly better than this.

In $\S 5$ we let $L$ be an Engel- $n$ Lie algebra over a field $K$ with $\operatorname{char}(K)>n$, and we let $x$ be an element of $L$. In Lemma 5 we considered a product

$$
v=x a_{11} a_{12} \cdots a_{1 k_{1}} x a_{21} a_{22} \cdots a_{2 k_{2}} x \cdots x a_{m 1} a_{m 2} \cdots a_{m k_{m}}
$$

of type $(m ; k)$ in $L$. We let $Y$ be the ideal of $L$ generated by elements of the form $x a_{1} a_{2} \cdots a_{n-1}$ where $a_{i} \in\left\{a_{r s} \mid 1 \leq r \leq m, 1 \leq s \leq k_{r}\right\}$ for $i=1,2, \ldots, n-1$, and we let $Z$ be the ideal of $L$ generated by all products in $L$ of type $(m ; r)$ with $r<k$. We showed that if $k \geq m+2+\frac{(m-1)(3 m-10)}{3}$ then $v \in Y+Z$. In the proof we made a number of inductive hypotheses, and we showed that these implied that $v=0$ unless

$$
\begin{gathered}
k_{1} \leq n-2, \\
k_{r} \leq n-3 \text { for } r=1,2, \ldots, m-1, \\
k_{m}=0, \\
k_{1}+k_{2}+k_{3} \leq 3 n-9, \\
k_{r}+k_{r+1}+k_{r+2} \leq 3 n-10 \text { for } r=2,3, \ldots, m-3 .
\end{gathered}
$$

I have used the nilpotent quotient algorithm for graded Lie rings to show that when $n=6$ and $\operatorname{char}(K)=7$ then the inductive hypotheses used in the proof of Lemma 5 also imply that $v=0$ if $k_{r}=2, k_{r+1}=3, k_{r+2}=3$ for some $r$ with $1<r<m-2$. This, together with the inequalities above, imply that $v \in Y+Z$ if $k>\frac{7 m-1}{2}$. We can then use the same argument as in the proof of Lemma 6 to show that if $x$ is an element of an Engel-6 Lie algebra of characteristic 
7 then $\left(I d_{L}(x)\right)^{55}$ is generated by products of type $(r ; k)$ with $19 \leq r \leq 55$, where $k \leq 65$ when $r=19$, and where $k \leq \frac{7 r-1}{2}$ when $20 \leq r \leq 55$. Then using the same argument as in the proof of Theorem 3, and taking $N=10$, we see that if $a, b \in L$ then $I d_{L}\left(a b^{4}\right)$ has class at most 54 .

Now let $L$ be the (relatively) free $m$-generator Engel-6 Lie algebra of characteristic 7. By Kostrikin's theorem [8], $L$ is nilpotent. Let $I$ be the ideal of $L$ generated by $\left\{a b^{4} \mid a, b \in L\right\}$. Traustason [13] has shown that $L / I$ is nilpotent of class 7 , which implies that $L^{8} \leq I$. Now let $S$ be a subset of the generators of $L$, let $L_{S}$ be the subalgebra of $L$ generated by $S$, and let $I_{S}$ be the ideal of $L$ generated by $\left\{a b^{4} \mid a, b \in L_{S}\right\}$. Then any product of eight elements from $S$ must lie in $I_{S}$. This implies that $L^{8} \leq \sum I_{S} \leq I$, where the sum is taken over all subsets $S$ of the free generators of $L$ such that $|S| \leq 8$. In fact $\sum I_{S}=I$, but we do not need to use this fact. I used the nilpotent quotient algorithm for graded Lie rings to compute the number of elements $a b^{4}$ which are needed to generate $I_{S}$ for $2 \leq|S| \leq 8$, and the computations showed that $\sum I_{S}$ can be generated by $d_{m}$ elements, where

$$
\begin{aligned}
d_{m}= & 322\left(\begin{array}{c}
m \\
8
\end{array}\right)+1197\left(\begin{array}{c}
m \\
7
\end{array}\right)+1725\left(\begin{array}{c}
m \\
6
\end{array}\right) \\
& +1219\left(\begin{array}{c}
m \\
5
\end{array}\right)+441\left(\begin{array}{c}
m \\
4
\end{array}\right)+153\left(\begin{array}{c}
m \\
3
\end{array}\right)+6\left(\begin{array}{c}
m \\
2
\end{array}\right) .
\end{aligned}
$$

Since each element $a b^{4}$ generates an ideal which is nilpotent of class at most 54 , it follows that $\sum I_{S}$ is nilpotent of class at most $54 d_{m}$. Since $L^{8} \leq \sum I_{S}$, we see that $L^{8}$ is also nilpotent of class at most $54 d_{m}$. Now if $J$ is any ideal of $L$, then

$$
J \underbrace{L L \cdots L}_{36} \leq J\left(L^{8}\right)
$$

(For a proof of this see, for example, Proposition 4.6 of Chapter 1 of [9].) It follows that $L^{8+36 k} \leq\left(L^{8}\right)^{k+1}$ for $k=1,2, \ldots$, and so the fact that $L^{8}$ is nilpotent of class at most $54 d_{m}$ implies that $L$ is nilpotent of class at most $7+1944 d_{m}$. Now $7+1944 d_{m}$ is a polynomial of degree 8 in $m$, with leading coefficient 15.525 . It is a straightforward calculation to show that $7+1944 d_{m}<$ $51 \mathrm{~m}^{8}$ for all values of $m$. This proves Theorem 7 .

\section{REFERENCES}

1. S. I. Adjan and A. A. Razborov, Periodic groups and Lie algebras, Uspekhi Mat. Nauk $\mathbf{4 2}$ (1987), 3-68.

2. N. D. Gupta and M. F. Newman, The nilpotency class of finitely generated groups of exponent four, Lecture Notes in Math., vol. 372, Springer-Verlag, Berlin, 1974, pp. 330-332.

3. P. Hall and G. Higman, On the p-length of p-soluble groups and reduction theorems for Burnside's problem, Proc. London Math. Soc. 6 (1956), 1-42.

4. G. Havas, M. F. Newman, and M. R. Vaughan-Lee, A nilpotent quotient algorithm for graded Lie rings, J. Symbolic Comput. 9 (1990), 653-664.

5. G. Havas, G. E. Wall, and J. W. Wamsley, The two generator restricted Burnside group of exponent five, Bull. Austral. Math. Soc. 10 (1974), 459-470.

6. P. J. Higgins, Lie rings satisfying the Engel condition, Proc. Cambridge Philos. Soc. 50 (1954), 8-15.

7. G. Higman, On finite groups of exponent five, Proc. Cambridge Philos. Soc. 52 (1956), 381-390. 
8. A. I. Kostrikin, The Burnside problem, Izv. Akad. Nauk SSSR Ser. Mat. 23 (1959), 3-34.

9. —_ Around Burnside (translated by J. Wiegold), Ergeb. Math. Grenzgeb., SpringerVerlag, Berlin, 1990.

10. F. Levi and B. L. van der Waerden, Über eine besondere Klasse von Gruppen, Abh. Math. Sem. Univ. Hamburg 9 (1933), 154-158.

11. Ju. P. Razmyslov, On a problem of Hall-Higman, Izv. Akad. Nauk SSSR Ser. Mat. 42 (1978), 833-847.

12. I. N. Sanov, Solution of Burnside's problem for exponent four, Leningrad State Univ. Ann. Math. Ser. 10 (1940), 166-170.

13. G. Traustason, Engel Lie algebras, Quart. J. Math. (2) 44 (1993), 355-384.

14. __ Engel Lie algebras, D. Phil. thesis, Oxford Univ., 1993.

15. M. R. Vaughan-Lee, Lie rings of groups of prime exponent, J. Austral. Math. Soc. 49 (1990), 386-398.

16. _ The restricted Burnside problem, Oxford Univ. Press, 1990.

17. M. R. Vaughan-Lee and E. I. Zelmanov, Upper bounds in the restricted Burnside problem, J. Algebra 161 (1993).

18. E. I. Zelmanov, The solution of the restricted Burnside problem for groups of odd exponent, Izv. Math. USSR 36 (1991), 41-60.

19. 568-592.

Christ Church, OXford, OX1 1DP, England

E-mail address: vlee@math.ox.ac.uk 